\title{
A study of heat distribution in human skin: use of Infrared Thermography
}

\author{
Domoina Ratovoson, Franck Jourdan ${ }^{\mathrm{a}}$, Vincent Huon
}

\author{
Laboratory of Mechanics and Civil Engineering \\ CNRS, Université de Montpellier 2 \\ Place Eugène Bataillon \\ 34095 Montpellier, France
}

\begin{abstract}
One of the long-term objectives of this study is to be able to act quickly on body burns, to avoid propagating lesions due to heat diffusion. An experimental and numerical study is presented to better understand the thermomechanical behavior of the skin and its direct environment when exposed to strong thermal variations. The experimental step, suggested in this article, consists in placing a cooled cylindrical steel bar on the skin of a human forearm and measuring the temperature change using an infra-red camera. Blood circulation in the veins was seen to clearly influence of the heat diffusion. These experimental measurements provide a numerical model of the skin and its direct vicinity. This two-dimensional multi-layer model uses the Pennes equation to model biological tissue and the equation of heat in a fluid, to model blood. The properties of materials from the literature are validated by experimentation. The numerical model is able to simulate the experimental observations, but also to estimate blood speed in the veins.
\end{abstract}

\section{Introduction}

The first works on thermal modeling of the skin date from the beginning of the $19^{e}$ century, but quickly collide with the complexity of its structure. Skin is a multilayer, living material. Blood activity plays a dominant role in heat distribution. With current experimental techniques (Doppler effects, infrared thermal imaging), observations have become more refined. This study quantitatively highlights certain effects of blood circulation on the diffusion of temperature. In the manner of studies undertaken by [1] and [2], an infrared camera was used to measure the change in temperature of the surface of the skin of a human forearm. Infrared thermography is a nondestructive technique giving highly accurate access to the temperature of surface. The originality of our study is that we vary blood circulation by using the effects of gravity. This approach allows us to quantify the temperature variations due to the speed of blood circulation. An attempt is also made to develop a numerical model of skin, which can both report observed phenomena and be predictive. In this field, the model generally used is that of Pennes, proposed in 1948 [3]. The latter, often criticized for its oversimplified nature, was enriched by Wulff [4] and Klinger [5], then re-examined by Chen and Holmes [7]. Their model highlights the faults of the Pennes model by taking account of the directional nature of blood flow and by incorporating the convective aspect of heat transfer by blood flow. Chen and Holmes developed a model of the heating effects of the various blood vessels according to architecture, geometry and blood flow characteristics. This model is more representative of reality than that of Pennes but it contains terms that appear inaccessible. For this reason the Pennes model was chosen for the present study The same choice was

a Corresponding author: Address: Laboratoire de Mécanique et Génie Civil, CC48, Université de Montpellier 2, Place Eugène Bataillon, 34095 Montpellier, France; phone: 33467149633; fax: 33467143923; email:jourdan@lmgc .univ-montp2.fr

This is an Open Access article distributed under the terms of the Creative Commons Attribution-Noncommercial License 3.0, which permits unrestricted use, distribution, and reproduction in any noncommercial medium, provided the original work is properly cited. 
made in the recent works of [8] and [9]. Our work is distinguished from the others by the modeling of blood circulation and the diffusion of heat around the veins. In this article, the experiments are first described, followed by the experimentation protocol and the material used, presented in detail. The results obtained with the infrared camera are then analyzed and compared. The thermomechanical multi-layer model is then developed with details of the method of resolution. The results of the numerical simulations are analyzed and the results of the experiment are compared with numerical calculations for the cooling and warming.

\section{Experimental device}

\subsection{Experimental protocol}

The aim of the experiment was to measure the evolution of temperature in vivo of the skin of the forearm. A cylindrical steel bar was used, previously cooled in a deep freeze for study the warming of the skin or warmed in a "four" for study the cooling of the skin. This bar was placed on the forearm of a 28 year-old woman and the change in temperature was measured using the infrared camera. During measurements, the temperature of the experimentation room was maintained at $20^{\circ} \mathrm{C}$. The camera was placed at approximately $50 \mathrm{~cm}$ from the points of measurement. When the bar was placed on the skin, the measurements were restricted to a surface area of $(6 \times 10) \mathrm{cm}^{2}$. The cylinder of cooled steel, of a ray of $1 \mathrm{~cm}$, was placed for 40 seconds on the surface of the skin and then removed. At the same moment the thermal camera started recording for the duration of 90 seconds. The forearm must be immobilized to obtain exploitable results. Two positions of the forearm were treated: a horizontal position and a vertical position. Surface temperatures were measured at the same location. The purpose was to quantify the effect of blood circulation on heat transfer. For the analysis, the parts containing veins were located and faster heating was expected in those zones.

\subsection{Experimental results}

\section{Horizontal position}

For the analysis, points were selected on the image (see figure 1). Certain points were located under the cylinder (3, 4 and 5) and others outside it (1,2,6 and 7).

\section{Comments}

Points 1 and 2 were located on the passage of a vein. Their temperature dropped quickly to $27^{\circ} \mathrm{C}$ and returned to thermal balance. This shows the influence of venous blood in temperature diffusion.

Points 3 and 4 were located under the cylinder with no vein passage. Their temperature rose normally versus time.

Point 5 was under the cylinder and on the passage of a vein. Its curve was shaped like those of points 3 and 4 but with a higher initial temperature.

Point 6 was outside the cylinder. There was little temperature variation.

Point 7 was at the periphery of the cylinder and on the passage of a vein. Its temperature varied like those of points 1 and 2, but more moderately.

\subsection{Comparison of experimental results: (horizontal position and vertical position)}

In order to compare heating speeds, the temperature measurements relating to the vertical and horizontal positions were recorded at the same checkpoint. To visualize the differences, the curves resulting from the horizontal and vertical positions were shifted in time so that the initial temperatures, at the checkpoint, were identical.

One notes in figure 2 that the skin heated more quickly in the horizontal position.

The following case was selected with the same principle of comparison, but for a checkpoint at the periphery of the cylinder.

These experimental results demonstrate, quantitatively, the effect of blood circulation on the thermal behavior of skin. These results will now be used to develop a predictive numerical model. 


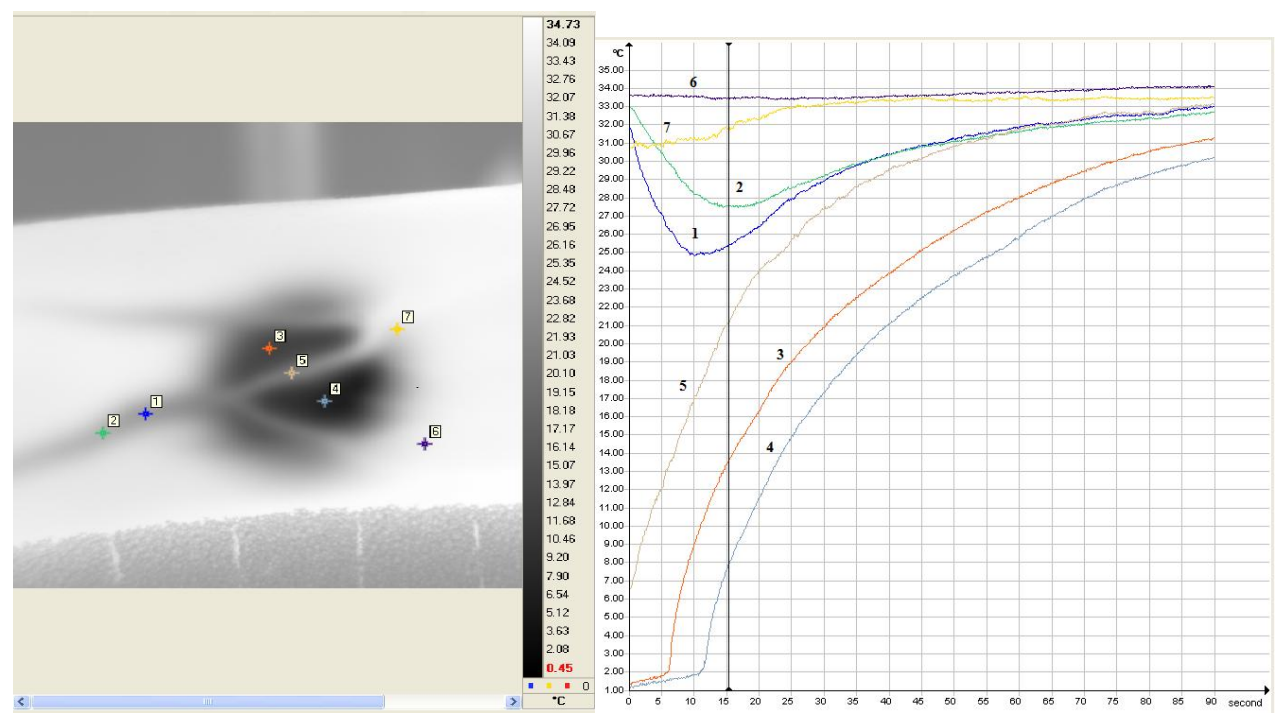

Fig. 1. Localization of checkpoints (left) and temperature of checkpoints (right) versus time (horizontal position)

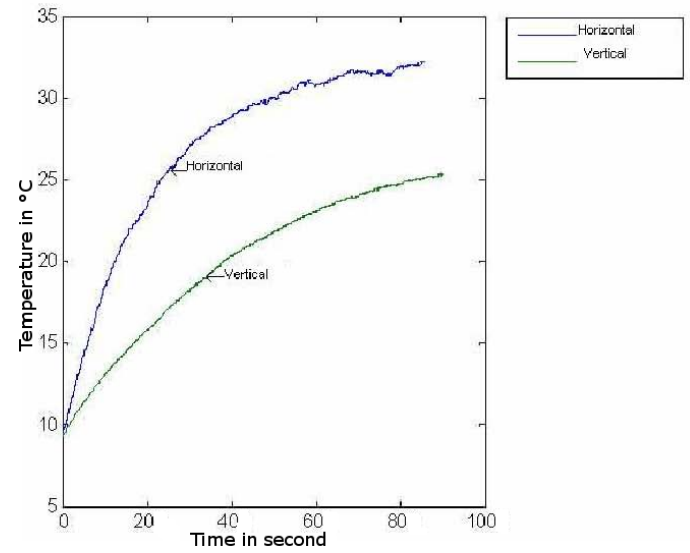

Fig. 2. Temperature of checkpoints under the cylinder: horizontal and vertical positions

\section{Modeling}

The model proposed in this paper is a bidimensional multi-layer model in which the veins are considered as fluid continuous media. The epidermis, the dermis and the hypodermis are considered as solid continuous media in which the evolution of heat is controlled by the Pennes equation.

\subsection{Heat transfer in a fluid continuous medium}

To model blood, the equations governing heat transfer in a fluid continuous medium are presented. A fluid is a continuous medium material that is deformable, without rigidity and which can run out. One notes $\underline{U}=\left(u_{1}, u_{2}, u_{3}\right)$ the velocity vector of a particle $P$ of position $\left(x_{1}, x_{2}, x_{3}\right)$ at time $t$. The strain rate tensor is defined by

$$
\epsilon_{i j}=\frac{1}{2}\left(\frac{\partial u_{i}}{\partial x_{j}}+\frac{\partial u_{j}}{\partial x_{i}}\right) \quad i, j=1,2,3
$$


The temperature is noted as $T$. Heat transfer in a homogeneous, incompressible and viscous fluid is controlled by the following partial derivative equation

$$
\rho C \frac{\partial T}{\partial t}-k \Delta T+\rho C \underline{U} \cdot \underline{\nabla} T=2 \mu \sum_{i=1}^{3} \sum_{j=1}^{3} \epsilon_{i j} \frac{\partial u_{i}}{\partial x_{j}}+r
$$

where $\rho$ is the specific mass $\left(\mathrm{Kg} \cdot \mathrm{m}^{-3}\right)$,

$C$ is the specific heat $\left(\mathrm{J} \cdot \mathrm{Kg}^{-1} \cdot{ }^{o} \mathrm{C}^{-1}\right)$,

$k$ is the thermal conductivity $\left(\mathrm{W} \cdot \mathrm{m}^{-1} \cdot{ }^{o} \mathrm{C}^{-1}\right)$,

$\mu$ is the viscosity (Pa.s),

$r$ is the heat source $\left(\mathrm{W} \cdot \mathrm{m}^{-3}\right)$. (neglected in our study)

\subsection{Pennes equation}

To model biologic tissues constituting the skin, the Pennes model was chosen. The first biothermal equation was developed by Pennes in 1948 [3]. There are two mechanisms characterizing heat transfer in living bodies: blood flow and metabolism. Before any study, and due to the varying size of the blood system, it is necessary to determine the scale to consider in studying heat transfers. The Pennes model proves to be most effective, with a scale ranging from a millimeter to several centimeters [10]. Pennes made the assumption that in a given volume of study, heat transfer $q_{p}$ between the blood and the tissue is proportional to the temperature difference between the arterial blood (entering the volume of study) and the (outgoing) venous blood.

$$
q_{p}=w(\rho C)_{b}\left(T_{a}-T_{v}\right)
$$

The index $b$ is reserved for blood coefficient of materials. The proportionality factor $w$ is called rate of perfusion. To connect the temperature of venous blood $T_{v}$ to the temperatures of arterial blood $T_{a}$ and of tissue $T_{t}$, Pennes introduced a coefficient $k^{\prime}$.

$$
T_{v}=T_{t}+k^{\prime}\left(T_{a}-T_{t}\right)
$$

For $k^{\prime}=0$, thermal balancing is complete, blood leaves tissue at the temperature $T_{t}$.

For $k^{\prime}=1$, there is no temperature variation of blood passing in the volume of study (blood leaves tissue at the arterial temperature).

The metabolism is also included in the biothermal equation as a distribution of energy source :

$$
Q_{m e t}=Q_{m e t}^{o} Q_{10}^{\frac{T_{o}-T}{10}}
$$

where $Q_{\text {met }}^{o}$ is the basal metabolism at $37^{\circ} \mathrm{C}, Q_{10}$ is the coefficient of temperature dependence ranging between 2 and $3 \mathrm{~W} \cdot \mathrm{m}^{-3}$ and $T_{o}$ is chosen at $37^{\circ} \mathrm{C}$.

The general biothermal equation obtained with the balance of energy for a volume of tissue taking account of the blood perfusion and the metabolism is given by :

$$
\rho C \frac{\partial T}{\partial t}-\underline{\nabla} k_{t} \cdot \underline{\nabla} T=w \rho_{b} C_{b}\left(T_{a}-T_{v}\right)+Q_{m e t}
$$

$\rho C \frac{\partial T}{\partial t}$ : Energy stored (W.m $\left.{ }^{-3}\right)$

$\underline{\nabla} k_{t} \cdot \underline{\nabla} T:$ heat conduction $\left(\mathrm{W} \cdot \mathrm{m}^{-3}\right) . k_{t}$ is the thermal conductivity of the tissue.

$\bar{w}(\rho \bar{C})_{b}\left(T_{a}-T_{v}\right):$ heat transport by perfusion $\left(\mathrm{W} \cdot \mathrm{m}^{-3}\right)$

$Q_{m e t}:$ generation of the metabolic internal power $\left(\mathrm{W} \cdot \mathrm{m}^{-3}\right)$.

For years, the Pennes model was criticized by some and defended by others. Despite these controversies and criticisms, most mathematical analyses carried out in biothermal transfers continue to be based on this equation [5], [11]. Let us note that these new models primarily enrich the modeling of heat transport by perfusion. The contribution of perfusion in heat transfers is treated like heat transfer in porous media. 


\subsection{Assumptions}

The model was composed of 3 layers, which are: the epidermis, the dermis and the hypodermis. Between the dermis and the hypodermis, blood circulation was created with a material having transport velocity. This material was given the properties of blood (see figure 3).

To resolve the problem, the following assumptions were made:

- The problem was treated in 2D.

- Only two layers (dermis and hypodermis) were taken into account. The epidermis was neglected because of its small thickness. Pennes equation was chosen to model heat transfer in tissue.

- $k_{t}$ was chosen constant, thus $\underline{\nabla} k_{t} \cdot \underline{\nabla} T=k \Delta T$.

- $k^{\prime}$ was chosen to be null, so as $T_{v}=T$.

- The generation of metabolic internal power $Q_{m e t}$ was taken as constant in the dermis and the hypodermis.

- A model of $30 \mathrm{~cm}$ length and $3 \mathrm{~mm}$ of thickness was used .

- For heat transfer in veins, equation (1) was used.

- Position and thickness of the vein are driven by parameters i1 and i2 .

- The velocity of the fluid was taken as constant and of direction $x_{1}$, the term $2 \mu \sum_{i=1}^{3} \sum_{j=1}^{3} \epsilon_{i j} \frac{\partial u_{i}}{\partial x_{j}}$ was then null.

- The properties of materials were collected in table 1 .

\begin{tabular}{|c|c|c|c|c|c|c|}
\hline $\begin{array}{c}\text { Sub-domain } \\
\text { number }\end{array}$ & Layer & $\begin{array}{c}\text { Thickness } \\
\text { (m) }\end{array}$ & $\begin{array}{c}\text { Specific } \\
\text { heat } \\
C\left(\mathrm{Jg}^{-1} .{ }^{o} \mathrm{C}^{-1}\right)\end{array}$ & $\begin{array}{c}\text { Thermal } \\
\text { conductivity } \\
k\left(\mathrm{~W} \cdot \mathrm{m}^{-1} \cdot{ }^{o} \mathrm{C}^{-1}\right)\end{array}$ & $\begin{array}{c}\text { Specific } \\
\text { mass } \\
\rho\left(\mathrm{Kg}^{-\mathrm{m}^{-3}}\right)\end{array}$ & $\begin{array}{c}\text { Rate } \\
\text { of perfusion } \\
w\left(\mathrm{~s}^{-1}\right)\end{array}$ \\
\hline 0 & Epidermis & $0,08 \cdot 10^{-3}$ & 3590 & 0,24 & 1200 & 0 \\
\hline 1 & Dermis & 0,002 & 3300 & 0,45 & 1200 & 0,00125 \\
\hline 2 & Blood & $0,001^{*}$ & 3770 & 0,45 & 1060 & 0 \\
\hline 3 & Hypodermis & 0,004 & 6500 & 0,69 & 2000 & 0,0025 \\
\hline
\end{tabular}

Table 1. Material properties [6], $0,001^{*}$ : the thickness of the veins can vary from 1 to $3 \mathrm{~mm}$

\subsection{Initial and boundary conditions}

Due to thermal profile of skin surface before cooled bar application, an initial temperature of $33^{\circ} \mathrm{C}$ was considered for the solid parts and of $35^{\circ} \mathrm{C}$ for blood. Calculations were carried out in two steps.

- Step 1 (duration 40s): A temperature $\mathrm{T}=35^{\circ} \mathrm{C}$ was imposed on the lower part of the hypodermis and at the entrance of the vein (on the left of figure 3). On a zone of $2 \mathrm{~cm}$ of the upper part of the dermis, the temperature was imposed at $1^{\circ} \mathrm{C}$ to model the action of the bar. The rest of the upper part of the dermis was under a condition of convection (see figure 3 ).

Convection was modeled by the Fourier's law :

$$
k \frac{\partial T}{\partial n}=h\left(T-T_{e}\right)
$$

where $n$ is the normal vector at the surface, $h$ is the coefficient of convection and $T_{e}$ is the temperature of air. We chose $h=7 \mathrm{~W} \cdot \mathrm{m}^{-2} \cdot{ }^{o} \mathrm{C}^{-1}$ (see [8]) and $T_{e}=20^{\circ} \mathrm{C}$.

- Step 2 (duration 90s): The same boundary conditions were imposed as in step 1, but the action of the bar was replaced by a condition of convection with air. Thus, all the upper part of the dermis was under convection condition. 


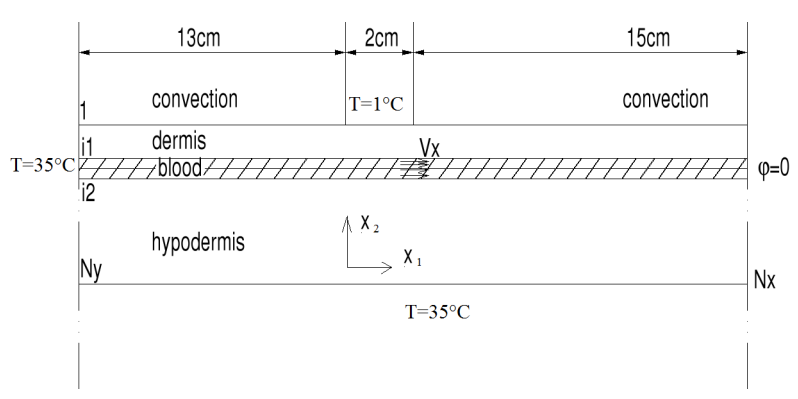

Fig. 3. Boundary conditions : Step 1

\section{Comparison between experimental and numerical results}

For the numerical resolution of the heat equation [3], the finite differences method was chosen and an explicit approach was adopted: the centered finite differences method. Time and space steps are not independent. They have been chosen to satisfy the CFL (Courant-Freidrichs-Lax) condition.

\subsection{Warming in vertical position}

To measure the differences between experimental and numerical results, three checkpoints were chosen. The three points were located on the passage of a vein (see figure 4). The first point was outside the cooled zone, downstream from the blood flow. The second point was under the cooled zone and the third point was outside this zone, but upstream of the blood flow. The generations of metabolic internal power in the dermis and the hypodermis were fixed at: $Q_{m e t}^{1}=Q_{m e t}^{3}=368 \mathrm{~W} \cdot \mathrm{m}^{-3}$ (see [12]).

The first series of comparisons was carried out on the horizontal forearm position. This fixed the parameters of the model and in particular blood velocity. The blood velocity was selected to best fit experimental data. The second series was carried out on the vertical position and validated the choice of parameters. It also allowed blood velocity variation to be estimated.

\section{Vertical position}

The checkpoints were the same as for the horizontal position. The comparison of the results is presented in the graph of figure 4.

As for the horizontal position, one observes the same shapes for experimental and numerical curves. For the numerical part, only the blood velocity was changed. It was fixed at $4.5 \mathrm{~mm} / \mathrm{s}$.

\subsection{Cooling and warming in horizontal position}

We made a comparative study of cooling and warming. We chose the check points in the center of the cooled zone and downstream from the blood flow. In this comparative study, we used a blood velocity of $4.8 \mathrm{~mm} / \mathrm{s}$. We observed a symmetric behavior between cooling and warming evolution (see figure $5)$.

\section{Comments and discussions :}

The numerical model reproduces experimental observations. It enables the properties of materials to be validated.

What should be retained from this study is that the numerical model is able to simulate the heat transport carried out by the blood flow. This particular point is shown by a drop followed by a fast rise in the temperature of tissue downstream from the cooled zone. This phenomenon is observed both experimentally and numerically.

Concerning blood velocity, a slight variation is seen between the horizontal and vertical positions in accordance with observations. This slight variation seems sufficient to modify the change of temperature in tissue and to reproduce the experimental observations. The value of $4.8 \mathrm{~mm} / \mathrm{s}$ may appear 

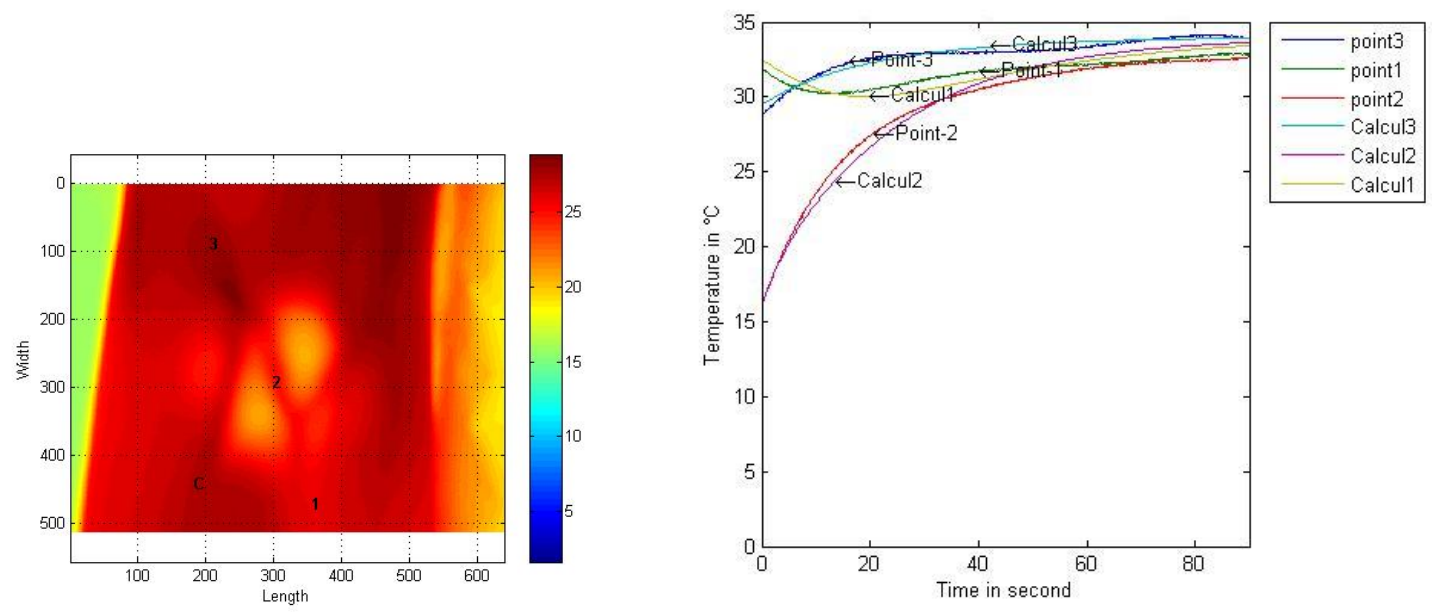

Fig. 4. Checkpoints - vertical position (left) / Comparison between experimental and numerical results for the vertical position (right)
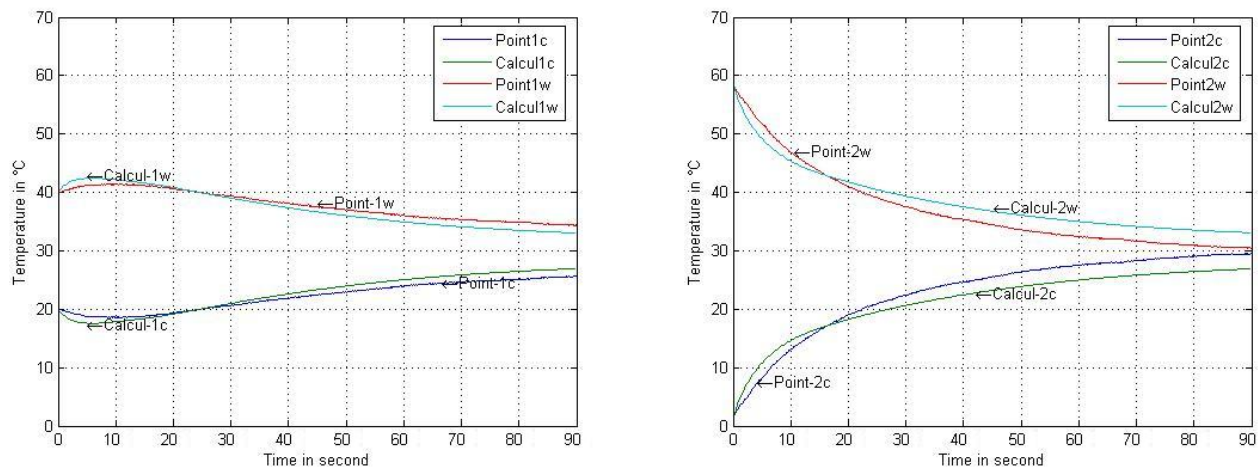

Fig. 5. Comparison between experimental and numerical results for cooling and warming check-point 1(left) /check-point 2 (right)

low compared to the work of Pennes [3], which shows velocities ranging from 3 to $6 \mathrm{~cm} / \mathrm{s}$. However, a similar study to ours [2] gives values between $0.16 \mathrm{~mm} / \mathrm{s}$ and $20 \mathrm{~mm} / \mathrm{s}$. According to the authors this variation depends on the individual.

The influence of metabolic power generation $Q_{m e t}$ and perfusion rate $w$ is not significant in the numerical results. For this reason no parametric study relative to $Q_{m e t}$ and $w$ was presented. Numerical observation confirms the works of [13], [14], [15] which suggest that the effects of the perfusion rate are significant in the case of long life thermal exposures and of low intensity or in the case of microwave exposure [16].

\section{Conclusion}

A thermal camera was used to record the temperature change of the skin of a human forearm, cooled during 40 seconds. The measurements highlighted complex phenomena. Certain effects of blood circulation on temperature diffusion were quantitatively observed. The influence of blood circulation was highlighted by comparing the temperatures obtained for a horizontal position and for a vertical position of the studied forearm. It was noted that the return to thermal balance was slower in the vertical 
position, which is justified by the effect of gravity on blood flow. Blood has more difficulty circulating in a vertical position than in a horizontal position.

In parallel, a numerical multi-layer model was developed, based on Pennes equation and able to simulate blood flow in the veins. This model can be used to validate the thermomechanical properties of the living materials constituting the various layers. It also provided an order of magnitude of blood velocity in the veins. This model confirms that the actions of blood perfusion and the metabolism are negligible for this kind of thermal stress.

Coupling between a nondestructive experimental approach and a numerical model, provides insight into the thermomechanical behavior of living tissue, as well as representing a promising approach. It offers a tool to explore the behavior of tissue exposed to high temperatures.

Experimentation at high temperature is not accessible in vivo in humans, which is why work will continue to be carried out by modeling. Finally, to take account of the directional effects, it is planned to prolong the two-dimensional model to a three-dimensional one.

\section{References}

1. H. Schnell, J. Zaspel, Cooling extensive burns: Sprayed coolants can improve initial cooling management: A thermography-based study, Burns, Volume 34, Issue 4, (2008) pp. 505-508.

2. C. Boué, F. Cassagne, C. Massoud, D.Fournier, Thermal imaging of a vein of the forearm: Analysis and thermal modelling, Infrared Physics \& Technology, Volume 51, Issue 1,(2007) pp. Pages 13-20.

3. H. H. Pennes, Analysis of tissue and arterial blood temperatures in resting human forearm, Journal of Applied Physiology, 1,(1948) pp. 93-122.

4. W. Wulff, The energy conservation equation for living tissue, IEEE Transactions on Biomedical Engineering, 36, (1974) pp. 403-415.

5. H. Klinger, Heat transfer in perfused biological tissue. i. general theory, Bull. Math. Biol., 21, (1974) pp. 494-497.

6. T. Gowrishankar, D. Stewart, G. Martin, J. Weaver, Transport lattice models of heat transport in skin with spatially heterogeneous, temperature-dependent perfusion, BioMedical Engineering OnLine, 3, (2004) p. 3:42.

7. M. Chen, K. Holmes, Microvascular contributions in tissue heat transfer, Annals of the New York Academy of Sciences, Vol. 335, (1980) pp. 137-150.

8. F. Xu, T. Lu, K. A. Seffen, Biothermomechanics of skin tissue, Journal of the Mechanics and Physics of Solids, 56 (5), (2008) p.18521884.

9. T. Shih, P. Yuan, W. Lin, H.Kou, Analytical analysis of the pennes bioheat transfer equation with sinusoidal heat flux condition on skin surface, Medical Engineering and Physics, 29, (2007) p. 946953.

10. S. Weinbaum, L. Jiji, A new simplified bioheat equation for the effect of blood flow on local average tissue temperature, ASME Trans. J. Biomech. Eng., 107, (1985) pp. 131-141.

11. S. Weinbaum, L. Jiji, D. Lemos, Theory and experiment for the effect of vascular temperature on surface tissue heat transfer - part i: Anatomical foundation and model conceptualization, ASME Journal of Biomedical Engineering, 106, (1984) pp. 321-330.

12. W. Roetzel, Y. Xuan, Transient response of the human limb to an external stimulustransient response of the human limb to an external stimulus, Int. J. Heat Mass Transfer, 41, (1998) pp. 229-239.

13. A. Shitzer, R. C. Eberhart, Heat transfer in medicine and biology: Analysis and application, vol 1 and 2., Plenum Press, New York (1985).

14. Z.-S. Deng, J. Liu, Parametric studies on the phase shift method to measure the blood perfusion of biological bodies, Med Eng Phys, 22, (2000) p. 693702.

15. L. Yong-Gang, L. Jing, Measurement of local tissue perfusion through a minimally invasive heating bead, Heat and Mass Transfer,Volume 44, Issue 2, (2007) pp. 201-211.

16. S. Ozen, S. Helhel, O. Cerezci, Heat analysis of biological tissue exposed to microwave by using thermal wave model of bio-heat transfer (twmbt), Burns, 34 (1), (2008) pp. 45-49. 